\title{
Synthesis and characterization of monosubstituted hexapyrrolylbenzene derivatives
}

\author{
Katarína Hrnčariková, ${ }^{a}$ Áron Szöllősy, ${ }^{\text {b and Daniel Végh }}{ }^{* a}$ \\ ${ }^{a}$ Department of Organic Chemistry, Slovak University of Technology, Radlinskeho 9, \\ SK-81237 Bratislava, Slovak Republic \\ ${ }^{b}$ Department of General and Analytical Chemistry, Technical University of Budapest \\ H-1521 Budapest, Hungary \\ E-mail: daniel.vegh@stuba.sk
}

(received 7 Jul 05; accepted 16 Dec 05; published on the web 11 Jan 06)

\begin{abstract}
Six new monosubstituted hexapyrrolylbenzene derivatives $\mathbf{3 a}-\mathbf{c}, \mathbf{4 a}-\mathbf{c}$ have been synthesized based on the multiple nucleophilic substitution reaction of appropriate 1-pentafluorophenyl-1Hpyrrole derivatives $1 \mathbf{a}-\mathbf{c}, \mathbf{2 a}-\mathbf{c}$ with the sodium salt of pyrrole in dry DMF.
\end{abstract}

Keywords: Propeller-shaped hexapyrrolylbenzenes, $\mathrm{S}_{\mathrm{N}} \mathrm{Ar}$ reaction, $\pi$-conjugation

\section{Introduction}

It is well known that oligomers and polymers with extended $\pi$-conjugation serve as important active components of electronic and opto-electronic devices. ${ }^{1}$ Currently a growing interest has been directed towards the design of molecular wires and rods, since such structures may serve as building blocks for nanoscale chemical entities that are geometrically and dimensionally confined. They may act as connectors in molecular and supramolecular electronic and photonic devices. The interest in chemically functionalized conductive materials continues to expand and currently encompasses electronic and opto-electronic devices, energy storage systems, bioelectrochemical sensors etc. Detecting single molecule properties, ${ }^{2,3}$ however, leads to completely new functional concept, and benzene-based compounds are particularly well-suited subjects for this developing technology. In these compounds is a synergism through which spectroscopic techniques are improved and in the course greater understanding of structureproperty relationships is gained. ${ }^{4}$ The study of stereochemistry of molecules is a relatively mature field. ${ }^{5}$

Highly symmetrical, propeller-shaped, hexasubstituted benzenes have received considerable interest due to their intriguing properties as sterically congested polycyclic aromatic compounds. ${ }^{6}$ Any aromaticity disscusion will soon rise the awareness that benzene chemistry is 
a starting point for polymer chemistry and in turn, that $\pi$-conjugated polymers contribute tremendously to theories of $\pi$-bonding structures. ${ }^{7,8}$ While the electronic design of conjugated polymers will appear to be an important concern below, it should be mentioned that benzenebased polymers should be looked at not only from the point of view of extended $\pi$-conjugation. Other important aspects of introducing benzene-based units into polymers are the high chemical and thermal stability of the building block and their easy chemical functionalization ${ }^{9}$ with all of the additional properties brought in by adding an appropriate group.

Pyrroles are very often used as a conducting interface because the substitution chemistry of the monomer is well defined and chemical and electrochemical polymerization is easy and the resulting polymers form robust and even films.

Monosubstituted hexapyrrolylbenzenes ${ }^{6,10}$ are unknown. Electrophilic substitution reactions of hexapyrrolylbenzene such as Vilsmeyer-Haack formylation and electrophilic acylation failed to lead to the formation of monosubstituted derivatives $\mathbf{3 a}-\mathbf{c}, \mathbf{4 a}-\mathbf{c}$.

\section{Results and Discussion}

The 2-substituted products $1 \mathbf{1 a}-\mathbf{c}$ could be selectively converted with high yields into the 3substutited products $\mathbf{2 a}-\mathbf{c}$ by treatment with trifluoromethanesulphonic acid. ${ }^{11}$ We now tried new synthetic route to $\mathbf{3 a}-\mathbf{c}, \mathbf{4 a}-\mathbf{c}$ starting from monosubstituted derivatives of 1-pentafluorophenyl$1 H$-pyrrole $1 \mathbf{a}-\mathbf{c}$ and $\mathbf{2 a}-\mathbf{c}$ by $\mathrm{S}_{\mathrm{N}} \mathrm{Ar}$ reaction with pyrrole sodium salt. The one-pot synthesis of compounds 3a-c, $\mathbf{4 a}-\mathbf{c}$ is based on the multiple nucleophilic substitution of the pentafluorophenyl group with 7.0 equivalents of pyrrolyl sodium salt in DMF (Scheme 1).

Since it is known that the rate of an $\mathrm{S}_{\mathrm{N}} \mathrm{Ar}$ reaction increases by the use of a more polar aprotic solvent, we performed the reaction of sodium salt of pyrrole with 1a-c and 2a-c in dry DMF at ambient temperature.

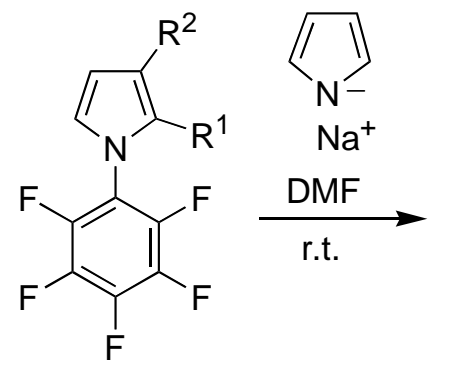

1,2

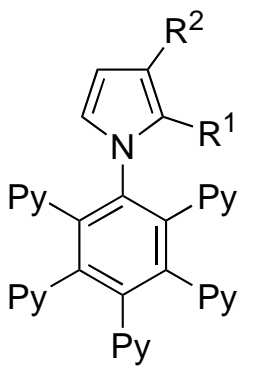

3, 4

\begin{tabular}{c|ll}
$\mathbf{1 , 3}$ & $\mathrm{R}^{1}$ & $\mathrm{R}^{2}$ \\
\hline $\mathbf{a}$ & $\mathrm{HCO}$ & $\mathrm{H}$ \\
$\mathbf{b}$ & $\mathrm{MeCO}$ & $\mathrm{H}$ \\
$\mathbf{c}$ & $\mathrm{EtCO}$ & $\mathrm{H}$
\end{tabular}

\begin{tabular}{c|ll} 
2, 4 & $\mathrm{R}^{1}$ & $\mathrm{R}^{2}$ \\
\hline $\mathbf{a}$ & $\mathrm{H}$ & $\mathrm{HCO}$ \\
$\mathbf{b}$ & $\mathrm{H}$ & $\mathrm{MeCO}$ \\
$\mathbf{c}$ & $\mathrm{H}$ & $\mathrm{EtCO}$
\end{tabular}

Py $=1 H$-pyrrol-1-yl

Scheme 1. Preparation of hexapyrrolylbenzenes 3 and 4. 
Mono $\alpha$-derivatives $\mathbf{1 a}-\mathbf{c}$ of hexapyrrolylbenzene afforded of requested products $\mathbf{3 a}-\mathbf{c}$ only in low yields. In our opinion this is due to steric hindrance by the substituent in $\alpha$-position of pyrrole. This suggestion was confirmed by $\mathrm{S}_{\mathrm{N}} \mathrm{Ar}$ of mono $\beta$-derivative $\mathbf{2 a}-\mathbf{c}$, which gave the corresponding $\mathbf{4 a}-\mathbf{c}$ in much higher yields. In both of cases the products were separated by column chromatography. Physical and chemical properties of $\mathbf{3 a}-\mathbf{c}$ and $\mathbf{4 a - c}$ compounds are collected in Table 1.

Table 1. Selected physical data and yields of $\mathbf{3 a}-\mathbf{c}$ and $\mathbf{4 a -}-\mathbf{c}$

\begin{tabular}{clllllc}
\hline Product & $\mathrm{R}$ & Eluant $^{\mathrm{a}}$ & $\mathrm{R}_{f}$ & $\mathrm{mp}\left[{ }^{\circ} \mathrm{C}\right]$ & color & Yield $^{\mathrm{b}}[\%]$ \\
\hline $\mathbf{3 a}$ & $\mathrm{H}$ & toluene & 0.07 & $300-303$ & colorless & 7 \\
$\mathbf{3 b}$ & $\mathrm{Me}$ & toluene & 0.11 & $253-254$ & yellow & 6 \\
$\mathbf{3 c}$ & $\mathrm{Et}$ & toluene & 0.11 & $208-212$ & yellow & 8 \\
$\mathbf{4 a}$ & $\mathrm{H}$ & toluene/EtOAc 20:1 & 0.31 & $209-215$ & light yellow & 39 \\
$\mathbf{4 b}$ & $\mathrm{Me}$ & toluene/EtOAc 20:1 & 0.13 & $271-272$ & light yellow & 55 \\
$\mathbf{4 c}$ & $\mathrm{Et}$ & toluene/EtOAc 20:1 & 0.21 & $239-240$ & light yellow & 47 \\
\hline
\end{tabular}

${ }^{a}$ Eluant used for column chromatography. ${ }^{b}$ Isolated yield after column chromatography.

We were not able to identify any by-products due to the difficult separation of the crude $\mathrm{S}_{\mathrm{N}} \mathrm{Ar}$ reaction mixtures. For confirmation of any atom of fluorine appears in desired products 3a-c and 4a-c, ${ }^{19}$ F NMR spectroscopy was utilized.

\section{Conclusions}

We have presented a facile and convenient synthetic method for the synthesis of monosubstituted hexapyrrolylbenzenes. Due to their sterically congested structures they may be regarded as building blocks for larger $\pi$-conjugated systems possessing propeller-shaped molecules with interesting optoelectronic properties.

Products 3a-c and $\mathbf{4 a}-\mathbf{c}$ were synthesized in order to study their conductive properties. We observed interesting results which will published as part of a full paper.

\section{Acknowledgments}

This work was financially supported by grants from the Ministry of Education of the Slovak Republic No. 1/1379/04 and the Science and Technology Assistance Agency (No. APVT-20007304) 


\section{Experimental Section}

General Procedures. Derivatives 1a-c and $\mathbf{2 a - c}$ were prepared according to the literature. ${ }^{11} \mathrm{~A}$ $60 \%$ dispersion of sodium hydride in mineral oil was used after removal of mineral oils with hexane. DMF was distilled at reduced pressure and kept in a dark bottle over $4 \AA$ molecular sieves. Pyrrole was used after distillation under reduced pressure and kept in a freezer. Thin layer chromatography (TLC) was carried out on silica gel $60 \mathrm{~F}_{254}$ (Merck) and ALUGRAM ${ }^{\circledR}$ SIL UV (Macherey-Nagel). All products were separated by column chromatography packed with silica gel 60 (0.040-0.063 mm, Merck). The ${ }^{1} \mathrm{H}$ and ${ }^{13} \mathrm{C}$ NMR spectra (DMSO- $d_{6}$ solutions) were recorded either with a Varian VXR-300 spectrometer (operating frequencies $295.0 \mathrm{MHz}$ for ${ }^{1} \mathrm{H}$ and $75.12 \mathrm{MHz}$ for ${ }^{13} \mathrm{C}$ ) or with a Bruker DRX-500 instrument (operating frequencies 500.13 $\mathrm{MHz}$ for ${ }^{1} \mathrm{H}$ and $125.76 \mathrm{MHz}$ for ${ }^{13} \mathrm{C}$ ). Mass spectra (AEI) were measured with a GC/MS 25 RFA instrument (Kratos Analytical, Manchester) equipped with a direct inlet system at $70 \mathrm{eV}$, trap current $100 \mu \mathrm{A}$ at temperature of the ion source $200{ }^{\circ} \mathrm{C}$. IR spectra ( $\mathrm{KBr}$ pellets) were measured on spectrometer PU 9800 FTIR and Impact 400 (Nicolet). Melting points were measured on a Kofler block.

\section{1-[2,3,4,5,6-Penta(1H-pyrrol-1-yl)phenyl]-1H-pyrrole-2-carbaldehyde $\quad$ (3a). Typical procedure}

In a $25 \mathrm{~mL}$ two-necked flask under an argon atmosphere the mixture of $\mathrm{NaH}(1.03 \mathrm{~g}, 27.0 \mathrm{mmol})$ and DMF $(30 \mathrm{~mL})$ was stirred, and pyrrole $(1.80 \mathrm{~g}, 27.0 \mathrm{mmol})$ was added through a dropping funnel. After the liberation of $\mathrm{H}_{2}$ had ceased the reaction mixture was stirred for $130 \mathrm{~min}$. and the solution of 1-penta(1H-pyrrol-1-yl)phenyl-1H-pyrrol-2-carbaldehyde (1a; $1.0 \mathrm{~g}, 3.83 \mathrm{mmol})$ in DMF $(10 \mathrm{~mL})$ was added dropwise within $15 \mathrm{~min}$. The reaction mixture was stirred for $24 \mathrm{~h}$ before it was poured into iced water $(100 \mathrm{~mL})$. The colorless precipitate formed was filtered off, washed with water $(5 \times 30 \mathrm{~mL})$ and air dried overnight. Separation by column chromatography on silica gel (toluene) afforded the colorless product 3a. ${ }^{1} \mathrm{H}$ NMR $\left(300 \mathrm{MHz}, \mathrm{DMSO}-d_{6}\right): \delta 9.23$ $(1 \mathrm{H}, \mathrm{s}), 7.04(1 \mathrm{H}, \mathrm{s}), 6.89(1 \mathrm{H}, \mathrm{s}), 6.31-6.21(10 \mathrm{H}, \mathrm{m}), 5.90-5.86(11 \mathrm{H}, \mathrm{m}) .{ }^{13} \mathrm{C}$ NMR $(75 \mathrm{MHz}$, DMSO-d $)_{6}: \delta 178.8,135.2,135.0,134.6,134.1,132.8,132.6,123.4,121.4,121.3,111.1,109.6$, 109.5, 109.2. MS: m/z (\%) 496 (100) $\left[\mathrm{M}^{+}\right], 469$ (17) [M-CHO], 404 (11) [M- $\mathrm{C}_{4} \mathrm{H}_{3} \mathrm{NCHO}, 77$ (6) $\left[\mathrm{C}_{6} \mathrm{H}_{5}\right]$. IR $(\mathrm{KBr}): \tilde{v} 3150,3100,1670,1550,1530,1510,1450 \mathrm{~cm}^{-1}$. Anal. Calcd for $\mathrm{C}_{31} \mathrm{H}_{24} \mathrm{~N}_{6} \mathrm{O}$ (496.20): C, 74.98; H, 4.87; N, 16.92. Found: C, 74.81; H, 4.85; N, 16.77;

Products $\mathbf{3 b}-\mathbf{c}$ and $\mathbf{4 a}-\mathbf{c}$ were prepared according to this procedure.

Selected physical data and yields: Table 1.

1-[2,3,4,5,6-Penta(1H-pyrrol-1-yl)phenyl)-1H-pyrrol-2-yl]ethanone $\quad(3 \mathrm{~b}) .{ }^{1} \mathrm{H} \quad \mathrm{NMR} \quad(300$ MHz, DMSO-d $)_{6}: \delta 6.90-6.88(2 \mathrm{H}, \mathrm{m}), 6.32(2 \mathrm{H}, \mathrm{m}), 6.26(4 \mathrm{H}, \mathrm{m}), 6.19(4 \mathrm{H}, \mathrm{m}), 6.10(1 \mathrm{H}, \mathrm{dd}$, $J=3.6,3.0 \mathrm{~Hz}), 5.92-5.89(6 \mathrm{H}, \mathrm{m}), 5.86(4 \mathrm{H}, \mathrm{m}), 2.07(3 \mathrm{H}, \mathrm{s}) .{ }^{13} \mathrm{C}$ NMR $\left(75 \mathrm{MHz}, \mathrm{DMSO}-d_{6}\right)$ : $\delta 178.2,135.5,134.7,134.5,134.4,132.2,130.9,121.4,121.3,121.2,119.7,110.1,109.5,109.1$, 26.4. MS: $m / z(\%) 510(100)\left[\mathrm{M}^{+}\right], 494$ (7) $\left[\mathrm{M}-\mathrm{CH}_{4}\right], 466(26)\left[\mathrm{M}-\mathrm{COCH}_{3}\right], 77$ (7) $\left[\mathrm{C}_{6} \mathrm{H}_{5}\right] . \mathrm{IR}$ 
$(\mathrm{KBr}): \tilde{v}$ 3150, 3100, 2950, 2810, 1660, 1550, 1540, 1510, $1440 \mathrm{~cm}^{-1}$. Anal. Calcd. for $\mathrm{C}_{32} \mathrm{H}_{26} \mathrm{~N}_{6} \mathrm{O}$ (510.20): C, 75.27; H, 5.13; N, 16.46. Found: C, 75.20; H, 5.10; N, 16.66.

1-[1-(2,3,4,5,6-Penta(1H-pyrrol-1-yl)phenyl)-1H-pyrrol-2-yl]propan-1-one (3c). ${ }^{1} \mathrm{H}$ NMR: (300 MHz, DMSO-d $\left.)_{6}\right): \delta .75-6.76(2 \mathrm{H}, \mathrm{m}), 6.20(2 \mathrm{H}, \mathrm{t}, J=2.1 \mathrm{~Hz}, \mathrm{~J}=1.8 \mathrm{~Hz}), 6.14(4 \mathrm{H}, \mathrm{m})$, 6.04-6.05 (4H, m), $5.96(1 \mathrm{H}, \mathrm{dd}, J=3.6 \mathrm{~Hz}, 3.0 \mathrm{~Hz}), 5.79-5.77(10 \mathrm{H}, \mathrm{m}), 2.32(2 \mathrm{H}, \mathrm{q}, J=7.2$ $\mathrm{Hz}), 0.76(3 \mathrm{H}, \mathrm{t}, J=7.2 \mathrm{~Hz}) .{ }^{13} \mathrm{C} \mathrm{NMR}\left(75 \mathrm{MHz}, \mathrm{DMSO}-d_{6}\right): \delta 190.7,135.6,134.8,134.5$, $134.4,131.9,130.7,121.5,121.4,121.3,119.5,118.8,110.1,109.6,109.2,31.1,8.2 . \mathrm{MS}: \mathrm{m} / \mathrm{z}$ (\%) 524 (100) $\left[\mathrm{M}^{+}\right], 495$ (13) [M- $\left.\mathrm{CH}_{2} \mathrm{CH}_{3}\right], 467$ (37) [M- $\left.\mathrm{COCH}_{2} \mathrm{CH}_{3}\right], 77$ (4) $\left[\mathrm{C}_{6} \mathrm{H}_{5}\right] . \mathrm{IR}(\mathrm{KBr})$ : $\tilde{v} 3130,3110,2980,2890,1665,1540,1520,1530,1440 \mathrm{~cm}^{-1}$. Anal. Calcd. for $\mathrm{C}_{33} \mathrm{H}_{28} \mathrm{~N}_{6} \mathrm{O}$ (524.20): C, 75.55; H, 5.38; N, 16.02. Found: C, 75.30; H, 5.18; N, 16.12.

1-[2,3,4,5,6-Penta(1H-pyrrol-1-yl)phenyl]-1H-pyrrol-3-carbaldehyde (4a). ${ }^{1} \mathrm{H}$ NMR (500 MHz, DMSO-d $\left.)_{6}\right): \delta 9.47(1 \mathrm{H}, \mathrm{s}), 7.17(1 \mathrm{H}$, broad s), 6.34-6.37 $(5 \mathrm{H}, \mathrm{m}), 6.26-6.29(7 \mathrm{H}, \mathrm{m})$, 5.88-5.95 $(10 \mathrm{H}, \mathrm{m}) .{ }^{13} \mathrm{C}$ NMR $\left(125 \mathrm{MHz}, \mathrm{DMSO}-d_{6}\right): \delta 185.2,135.3,135.0,134.8,133.9$, $131.8,127.0,125.3,121.8,121.5,121.4,110.2$, 109.8, 109.7, 107.4. MS: m/z (\%) 496 (100) $\left[\mathrm{M}^{+}\right], 469(10)[\mathrm{M}-\mathrm{CHO}], 401(50)\left[\mathrm{M}-\mathrm{C}_{4} \mathrm{H}_{3} \mathrm{NCHO}\right], 77$ (10) $\left[\mathrm{C}_{6} \mathrm{H}_{5}\right] . \mathrm{IR}(\mathrm{KBr}): \tilde{v}$ 3150, 3100, 1650, 1550, 1480, 1520, $1500 \mathrm{~cm}^{-1}$. Anal. Calcd. for $\mathrm{C}_{31} \mathrm{H}_{24} \mathrm{~N}_{6} \mathrm{O}$ (496.20): C, 74.98; H, 4.87; N, 16.92. Found: C, 74.83; H, 4.83; N, 16.80 .

1-[1-(2,3,4,5,6-Penta(1H-pyrrol-1-yl)phenyl)-1H-pyrrol-3-yl]ethanone (4b). ${ }^{1} \mathrm{H}$ NMR (500 MHz, DMSO-d 6$): \delta 7.01(1 \mathrm{H}, \mathrm{dd}, J=1.7,1.7 \mathrm{~Hz}), 6.35-6.38(5 \mathrm{H}, \mathrm{m}), 6.26-6.30(7 \mathrm{H}, \mathrm{m}), 5,94-$ $5.96(4 \mathrm{H}, \mathrm{m}), 5.92-5.94(6 \mathrm{H}, \mathrm{m}),, 2.07(3 \mathrm{H}, \mathrm{s}) .{ }^{13} \mathrm{C}$ NMR $\left(125 \mathrm{MHz}, \mathrm{DMSO}-d_{6}\right): \delta 191.8,135.0$, $134.9,134.5,134.0,127.8,126.3,123.7,121.7,121.4,121.3,109.74,109.69,109.63,108.8$, 26.8. MS: $m / z(\%) 510(100)\left[\mathrm{M}^{+}\right], 495(15)\left[\mathrm{M}-\mathrm{CH}_{3}\right], 466(8)\left[\mathrm{M}-\mathrm{COCH}_{3}\right], 77$ (7) $\left[\mathrm{C}_{6} \mathrm{H}_{5}\right] . \mathrm{IR}$ (KBr): $\tilde{v}$ 3160, 3150, 2950, 2810, 1650, 1600, 1530, 1500, $1450 \mathrm{~cm}^{-1}$. Anal. Calcd. for $\mathrm{C}_{32} \mathrm{H}_{26} \mathrm{~N}_{6} \mathrm{O}$ (510.20): C, 75.27; H, 5.13; N, 16.46. Found: C, 75.22; H, 5.15; N, 16.70.

1-[1-(2,3,4,5,6-Penta(1H-pyrrol-1-yl)phenyl)-1H-pyrrol-3-yl]propan-1-one (4c). ${ }^{1} \mathrm{H}$ NMR $\left(500 \mathrm{MHz}, \mathrm{DMSO}-d_{6}\right): \delta 7.01(1 \mathrm{H}, \mathrm{dd}, J=1.9,1.9 \mathrm{~Hz}), 6.33-6.36(5 \mathrm{H}, \mathrm{m}), 6.25-6.29(7 \mathrm{H}, \mathrm{m})$, 5.92-5.95 (4H, m), 5.90-5.92 (6H, m) $2.45(2 \mathrm{H}, \mathrm{q}, J=7.2 \mathrm{~Hz}), 0.99(3 \mathrm{H}, \mathrm{t}, J=7.2 \mathrm{~Hz}) .{ }^{13} \mathrm{C}$ NMR (125 MHz, DMSO-d $)_{6}: \delta 195.2,135.1,134.9,134.5,134.0,127.3,125.6,123.7,121.7$, $121.5,121.4,109.74,109.68,108.8,31.9,8.7 . \mathrm{MS}: \mathrm{m} / \mathrm{z}(\%) 524$ (100) $\left[\mathrm{M}^{+}\right], 495$ (44) [M$\left.\mathrm{CH}_{2} \mathrm{CH}_{3}\right], 467$ (8) $\left[\mathrm{M}-\mathrm{COCH}_{2} \mathrm{CH}_{3}\right], 77$ (5) $\left[\mathrm{C}_{6} \mathrm{H}_{5}\right]$. IR (KBr): $\tilde{v}$ 3130, 3100, 3000, 2950, 1670, 1530, 1520, 1500, $1420 \mathrm{~cm}^{-1}$. Anal. Calcd. for $\mathrm{C}_{33} \mathrm{H}_{28} \mathrm{~N}_{6} \mathrm{O}$ (524.20): C, 75.55; H, 5.38; N, 16.02. Found: C, 75.33; H, 5.20; N, 16.10.

\section{References}

1. Kraft, A.; Grimsdale, A. C.; Holmes, A. B. Angew. Chem., Int. Ed. 1998, 37, 402; Angew. Chem. 1998, 110, 416. 
2. (a) Ambrose, W. P.; Goodwin, P. M.; Jett, H.; Van Orden, A.; Werner, J. H.; Keller, R. A. Chem. Rev. 1999, 99, 2929. (b) Tamarat, P.; Maali, A.; Lounis, B.; Orrit, M. J. Phys. Chem. A 2000, 104, 1.

3. Harada, Y.; Funatsu, T. Methodol. Cell Biol. 1998, 55, 117.

4. Watson, M. D.; Fechtenkötter, A.; Müllen, K. Chem. Rev. 2001, 101, 1267.

5. Nicolau, K. C.; Boddy, C. N. C.; Siegel, J. S. Angew. Chem., Int. Ed. 2001, 40, 701.

6. Biemans, A. M.; Zhang, C.; Smith, P.; Kooijman, H.; Smeets, W. J. J.; Spek, L.; Meijer, E. W. J. Org. Chem. 1996, 61, 9012.

7. Inoue, K.; Prog. Polym. Sci. 2000, 25, 453.

8. Soos, Z.G.; Mukhopadhyay, D.; Painelli, A.; Girlando, A. Handbook of Conducting Polymers, 2nd ed.; Marcel Dekker: New York, 1998.

9. Barber, J.; Siddigui, J. A. Abstr. Pap. Am. Chem. Soc. 1998, A215: U440, Pt2.

10. Henrie II, R. N.; Yeager, W. H. Heterocycles 1993, 61, 9012.

11. Hrncarikova, K.; Vegh, D. Molecules 2003, 536. 